\title{
Le nouveau registre des codes-créanciers (rcc): davantage d'informations, une meilleure répartition
}

P. Kraft

Le secteur de santé - et donc les rapports entre les médecins et les assureurs-maladie - ne cesse d'évoluer. La toute dernière innovation capitale qui mérite d'être signalée a été l'introduction du tarif pour les prestations ambulatoires TARMED. En réaction à ces changements, le registre des codes-créanciers (rcc), interface central entre fournisseurs de prestations et assureurs, se devait de réagir en se soumettant à une révision complète.

Le conseil d'administration de santésuisse a décidé le 29 octobre 2003 de regrouper les deux domaines Admissions et Mutations, qui étaient séparés jusqu'ici. Le domaine Admissions a été chargé d'élaborer et de mettre en œuvre un concept de révision du rcc. Le 27 janvier 2004, une première version a été approuvée, et le $1^{\mathrm{er}}$ juillet de la même année, la nouvelle application rcc est devenue opérationnelle à Lucerne sous la direction de Franz Wolfisberg, secrétaire général de santésuisse Suisse centrale et nouveau chef de ressort.

\section{Attribution optimale grâce à des indications détaillées}

Par rapport à l'ancienne version, le nouveau registre des codes-créanciers renferme des modifications et améliorations substantielles. Pour obtenir un numéro rcc, les fournisseurs de prestations doivent dorénavant fournir plus d'indications et de documents. Ainsi l'attribution par groupes de spécialité s'en trouve améliorée, d'autant plus que ces groupes ont été harmonisés avec ceux de la FMH, ce qui revêt une certaine importance par rapport à la procédure d'économicité: la comparaison des fournisseurs de prestations est plus pointue et les contrôles sont plus justes et plus efficaces. Pour les numéros rcc déjà existants, les nouvelles informations collectées doivent être saisies rétroactivement. Dans la mesure du possible, on s'est servi des banques de données actuelles. Cependant, les informations ainsi récoltées demandent à être vérifiées. De ce fait, des milliers de supports de données de four- nisseurs de prestations sont en train d'être traités dans le seul but de mettre à jour le registre des codes-créanciers. Autre mesure d'accompagnement: lors d'avis de mutation des fournisseurs de prestations, les données manquantes sont réclamées par l'intermédiaire d'un questionnaire.

\section{Exigences techniques élevées}

La nouvelle structure de la banque de données rcc a nécessité la mise à niveau du système informatique des assureurs-maladie. Comme il fallait s'y attendre, des difficultés notables sont apparues, de telle sorte que l'exploitation parallèle de l'ancienne et de la nouvelle structure sera poursuivie jusqu'à mi-2005. santésuisse veut éviter à tout prix que les assureurs se trouvent dans une «situation critique» au niveau des données et que, de ce fait, les décomptes de prestations deviennent plus difficiles.

La nouvelle version rcc ne contient pas un historique des données. Cela signifie que tous les documents originaux ayant servi à une attribution ou à une mutation d'un numéro rcc sont archivés. Pour éviter d'avoir recours à un archivage en salle, les documents sont numérisés et donc disponibles sur le champ. Il est prévu de rattacher directement les documents scannés à l'application rrc.

\section{Qu'est-ce qui change pour les médecins?}

Pour les médecins, l'harmonisation des désignations des groupes de spécialité avec ceux de la FMH représente un perfectionnement majeur. S'il n'y a pas de spécialité FMH ou s'il y en a plusieurs, on recherche l'activité de cabinet principale à laquelle le médecin doit être attribué. C'est pourquoi, dorénavant, le médecin qui demande un numéro rcc, doit fournir les renseignements suivants:

- diplôme suisse ou reconnu par la Confédération; 
- autorisation cantonale d'exercice de l'activité professionnelle avec admission à pratiquer à charge de l'assurance obligatoire des soins;

- justificatif du titre FMH ou du titre de formation continue FMH;

- déclaration d'adhésion à TARMED;

- justificatif de l'annonce faite à l'AVS comme indépendant.

Les données suivantes sont récoltées par l'intermédiaire d'un questionnaire:

- renseignements plus détaillées sur la personne;

- qualifications professionnelles complémentaires;

- numéro EAN;

- orientation du cabinet médical;

- installation du cabinet médical;

- activité de médecin agréé;

- conditions d'engagement;

- informations contractuelles et tarifaires ainsi que mention des opérations électroniques de paiement.
Nous invitons les médecins à signaler toutes sortes de mutation (nouvelle adresse, nouvelle orientation de cabinet, etc.) à l'adresse électronique zsr@santésuisse.ch. Pour des raisons de sécurité, les changements dans l'adresse de paiement (chèque postal, banque) nécessitent une notification écrite.

Pour conclure, nous vous signalons que tous les médecins employés, que ce soit dans les cabinets de groupe ou les cabinets individuels, doivent s'annoncer auprès du ressort rcc. En effet, cette catégorie de praticiens a également besoin d'une autorisation professionnelle de pratiquer de la part du canton pour pouvoir exercer à charge de l'assurance obligatoire des soins. Seuls les «assistants en formation» ne sont pas touchés par cette réglementation. Nous vous renvoyons à ce sujet aux articles 8 et 9 de la convention-cadre TARMED.

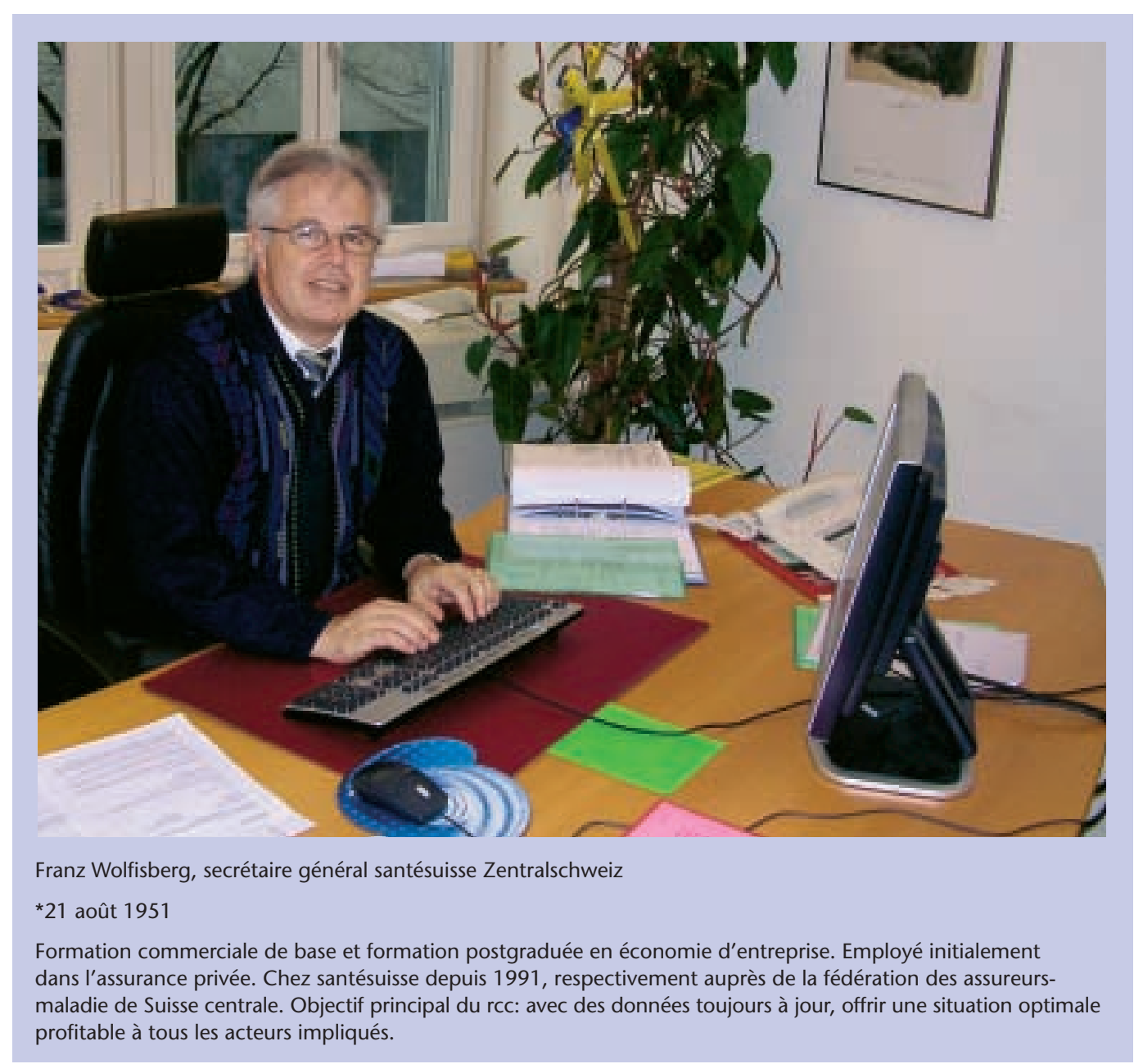

